\title{
Grip Strength as a Predictor of the Functional Outcome of Hip-Fracture Patients
}

\author{
RYUKI HASHIDA*, HIROO MATSUSE*, MASAFUMI BEKKI*, SOHEI IWANAGA*, \\ TAKAHIRO HIGUCHI**, YOHEI HIRAKAWA**, ASAMI KUBOTA ${ }^{\dagger}$,

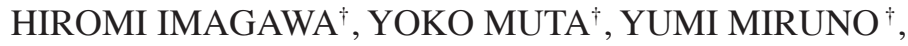 \\ KAZUHITO MINAMITANI** AND NAOTO SHIBA* \\ * Department of Orthopedics, Kurume University School of Medicine, Kurume 830-0011, \\ ** Department of Orthopedics, ${ }^{\dagger}$ Department of Nursing, \\ Munakata Suikokai General Hospital, Fukutsu 811-3298, Japan \\ Received 23 July 2019, accepted 2 October 2019 \\ J-STAGE advance publication 25 October 2021 \\ Edited by KIMIAKI SATO
}

\begin{abstract}
Summary: Background: Patients with hip fracture are limited as to physical activity. It is difficult to evaluate the physical function of the legs at admission; however, it is easy to measure grip strength, which has been reported to be correlated with systemic muscular strength and physical function. The objective of this study was to investigate the utility of grip strength in predicting functional outcome after hip fracture.

Methods: Fifty-seven patients who underwent surgery for hip fracture were evaluated for height, weight, Body Mass Index (BMI), grip strength, bone density (femoral neck), hemoglobin, Hemoglobin A1c (HbA1c), Hasegawa's Dementia Scale-Revised (HDS-R), and albumin at admission and Functional Independence Measure (FIM) at discharge. Spearman's rank correlation coefficient was used to evaluate the relation between grip strength and the above variables. Furthermore, factors of walking acquisition were analyzed by logistic regression analysis and decision-tree analysis.

Results: Correlation analysis showed that grip strength was positively correlated with bone density at admission and FIM at discharge and negatively correlated with age. In the logistic regression analysis, the independent factor associated with walking acquisition was grip strength (OR 1.26; 95\% CI 1.018-1.566; $\mathrm{p}=0.0339$ ). In the decisiontree analysis, grip strength was the initial divergence variable for walking acquisition (the percentage with walking acquisition was $80.0 \%$ of the patients with grip strength $\geqq 13.2 \mathrm{~kg}$ VS. $18.7 \%$ of the patients with grip strength $<$ $13.2 \mathrm{~kg})$.
\end{abstract}

Conclusions: Grip strength at admission was definitive in predicting the functional outcome of patients with hip fracture who underwent surgery.

Keywords grip strength, lower extremity strength, acute care hospital, functional prognosis

\section{INTRODUCTION}

The world's population is rapidly aging, which has resulted in an increase in the prevalence of many chronic diseases commonly seen in older patients [1].
Burge et al. estimated that 2 million fractures related to osteoporosis occurred in 2005 and projects that 3.5 million osteoporotic fractures will occur in 2025. The associated medical costs of these osteoporotic fractures were estimated to be $\$ 19$ billion in 2005, with an

Corresponding Author: Ryuki Hashida, M.D., Ph.D., Department of Orthopedics, Kurume University School of Medicine, 67 Asahi-machi, Kurume 8300011, Japan. Tel: +81-942-31-7568, Fax: +81-942-35-0709, E-mail: hashida_ryuuki@med.kurume-u.ac.jp 
expected increase to $\$ 25.3$ billion in 2025 [2]. Osteoporotic hip fracture is also associated with a high mortality rate, and is the most serious outcome of osteoporosis. Approximately $24 \%$ of hip fracture patients die in the year following their fracture [3]. In addition to the high mortality rate, hip fracture results in a need for nursing care due to impaired ADL (Activities of daily living), which is becoming problematic for aging societies.

Muscle strength is assessed by various techniques at different body locations. Grip strength is measured easily, and is used widely as a reliable and feasible surrogate for whole body strength [4]. Grip strength is especially related to lower extremity strength and cross-sectional muscle areas [5]. Moreover, grip strength is considered a marker of nutritional status and sarcopenia $[4,6]$. Of all muscle function tests, grip strength is an especially non-invasive marker of muscle strength and is well suited for clinical use.

Portegijs et al showed that lower extremity strength deficit affects mobility after proximal femoral fracture [7]. Although lower extremity muscular strength relates to walking ability, it is difficult to measure at the time of admission of femoral hip fracture patients. On the other hand, grip strength is the only physical function that can be easily measured at admission for these patients. Studies of the grip strength of hip fracture patients exist only for patients admitted to rehabilitation hospitals after surgery and show that grip strength is a good indicator of functional prognosis $[5,8]$. However, it remains unclear whether grip strength measured immediately after hip fracture in acute care facilities is predictive of functional outcome. If physical function could be accurately evaluated in the early stages of hospitalization and used to predict walking ability at the time of discharge, the rehabilitation target would become clearer and medical expenses would decrease.

The objective of this study was to investigate the utility of grip strength for predicting functional outcome after surgery for hip fracture.

\section{METHODS}

\section{Participants}

Between July 2015 and January 2017, fifty-seven patients with hip fracture were treated surgically at our hospital (Munakata Suikoukai General Hospital, Japan). All 57 fractures were either femoral cervical fracture or trochanteric fracture $(21 / 36)$.

The inclusion criteria were as follows: (1) 65 years of age or more, (2) underwent artificial femoral head replacement or internal fixation, and (3) weight bearing tolerance on the fractured hip.

The exclusion criteria were as follows: patients with (1) pathological fracture or open fracture (2) postoperative infection or a severe complication that did not allow initiation of weight bearing within two weeks after surgery, and (3) history of cranial nerve disease such as cerebral hemorrhage.

\section{Outcome Measures \\ Handgrip strength}

Handgrip strength was measured using a Smedleytype hand dynamometer (TKK5401; Takei Scientific Instruments Co., Ltd., Niigata, Japan) within three days after hospitalization with the patients in the supine position. The highest value of three assessments of the dominant hand was used for analysis. The assessment of grip strength has been shown to be reliable, with no difference between the sitting and supine positions [9].

\section{Laboratory determinations}

The following biochemical examination levels were measured using standard clinical methods: hemoglobin, albumin, and hemoglobin A1c.

\section{Bone mineral density}

Areal bone mineral density of the posterior-anterior lumbar spine was measured by dual-energy x-ray absorptiometry using a Hologic QDR 4500A densitometer (Hologic, Waltham, MA). All scans of an individual subject were performed on the same densitometer. Quality control measurements were performed daily with a Hologic anthropomorphic spine phantom.

\section{Isometric knee extension strength}

Isometric knee extension strength on the healthy side was measured at discharge using a manual muscle strength monitor (Mobie; Sakai Medical Co., Ltd.) [10]. Patients sat on the edge of a bed with their feet not touching the floor and with their arm crossed in front of the body. The highest value of three assessments was used in the analysis.

\section{FIM (Functional Independence Measure)}

The Functional Independence Measure (FIM) is a commonly used assessment based on observation by professionals who have been trained in its use. It is used to rate performance and degree of independence in everyday activities and thus to indicate how much assistance the patient will require in order to carry out ADL [11]. 


\section{Cognitive function}

Evaluation of cognition and screening for dementia were done using Hasegawa's Dementia Scale, Revised (HDS-R) [12]. This scale consists of nine items (age, orientation to time and date, orientation to place, memory of three words, calculation, recall of numbers in reverse order, delayed recall of three words, memory of five objects, and verbal fluency). Cognitive function was assessed using HDS-R (range, 0-30) at admission.

\section{Walking acquisition criteria}

Walking ability at discharge was evaluated according to the criteria for walking acquisition defined below. 1) Patients could walk for 10 meters with or without a T-cane at discharge. 2) Patients could walk for 10 meters by themselves without the assistance of a physical therapist.

\section{Rehabilitation protocol}

Patients did rehabilitation 1 to 2 hours a day for 6 days a week assisted by physical and occupational therapists. Our rehabilitation program included strength, range of motion, balance, and activities of daily living exercises.

\section{Statistical analysis}

Data are expressed as the median (interquartile range (IQR)), range, or number. The Spearman's Rank Correlation Coefficient was used to evaluate the relation between handgrip strength and the variables. $\mathrm{Pa}$ tients who met the conditions mentioned above were defined as the walking acquisition group. Differences in handgrip strength, host factors and FIM between the walking acquisition and non-walking acquisition groups were analyzed with the Wilcoxson Rank Sum Test. Independent factors associated with the acquisition of walking were evaluated by multivariate stepwise analysis. In addition, a decision-tree analysis was performed to identify the profiles associated with the acquisition of walking as previously described [13]. The level of statistical significance was set at $\mathrm{P}<0.05$.

\section{RESULTS}

\section{Patient characteristics}

Overall, 57 patients fulfilled the inclusion criteria and were enrolled in the study. Six patients were excluded (three who did not undergo surgery, two hemiplegic patients, and one under 65 years old). Patient characteristics are summarized in Table 1. The median age was 86 years, and the female to male ratio was
45:12. The median height was $1.50(\mathrm{~m})$, median weight $42 \mathrm{~kg}$, and body mass index (BMI) $18.9 \mathrm{~kg} / \mathrm{m}^{2}$. The cervical to trochanteric hip fracture ratio was 21:36. The median grip strength of the dominant hand at admission was $12.3 \mathrm{~kg}$. The median grip strength in male patients at admission was $22.9 \mathrm{~kg}$. The median grip strength in female patients at admission was 10.4 $\mathrm{kg}$. The median level of hemoglobin, albumin, and Hemoglobin A1c (HbA1c) was $11.6(\mathrm{~g} / \mathrm{dL}), 3.4 \mathrm{~g} / \mathrm{dL}$, and $5.8 \%$, respectively. The median bone mineral density was $0.69\left(\mathrm{~g} / \mathrm{cm}^{2}\right)$. The median isometric knee extension strength on the healthy side at discharge was 5.9 (kgf). The median HDS-R on admission was 14. The median period of hospitalization was 65 days.

\section{Factors correlated with grip strength}

Grip strength was not significantly correlated with BMI, albumin, or Hemoglobin A1c (HbA1c) (Table 2). There was a significant positive correlation between grip strength and height, weight, hemoglobin, HDS-R at admission, bone mineral density, isometric knee extension strength on healthy side at discharge, period of hospitalization, and FIM at discharge. Meanwhile, a significant negative correlation was seen between grip strength and age (Table 2).

\section{Difference in grip strength, host factors, and FIM at discharge between the walking acquisition and non- walking acquisition groups}

Of the 57 patients, 25 were in the walking acquisition group at discharge. There was no significant difference between the walking acquisition and nonwalking acquisition groups in terms of hemoglobin, albumin, HbA1c, bone mineral density or sex ratio (Figure 1C, D, E, F, I). In contrast, grip strength, HDS$\mathrm{R}$ and FIM were significantly higher in the walking acquisition group than in the non-walking acquisition group (Figure 1A, G, H). Age was significantly lower in the walking acquisition group than in the non-walking acquisition group (Figure 1B).

\section{Independent factors for walking acquisition were ex- amined by multivariate analysis.}

Grip strength and HDS-R were extracted in the multivariate stepwise procedure. (Table 3 ). Independent factors for walking acquisition were grip strength and HDS-R (OR 1.26; 95\% CI 1.018-1.566; $\mathrm{P}=0.0339$, OR $1.13 ; 95 \%$ CI $1.0123-1.257 ; \mathrm{P}=0.0188)$.

\section{Decision-tree algorithm for walking acquisition}

In the decision-tree analysis, grip strength was selected as the initial split, with $80.0 \%$ of the patients 
TABLE 1.

Patient characteristics

\begin{tabular}{|c|c|c|c|}
\hline & $\begin{array}{c}\text { Reference } \\
\text { value }\end{array}$ & Median (IQR) & $\begin{array}{c}\text { Range } \\
\text { (min-max) }\end{array}$ \\
\hline $\mathrm{N}$ & $\mathrm{N} / \mathrm{A}$ & 57 & \\
\hline Age & $\mathrm{N} / \mathrm{A}$ & $86(83-91)$ & $68-98$ \\
\hline Sex (female/male) & N/A & $45 / 12$ & \\
\hline Height (m) & $\mathrm{N} / \mathrm{A}$ & $1.50(1.43-1.55)$ & $1.35-1.68$ \\
\hline Weight $(\mathrm{kg})$ & N/A & $42(37.1-49.1)$ & $30.1-64.0$ \\
\hline Body mass index $\left(\mathrm{kg} / \mathrm{m}^{2}\right)$ & N/A & $18.9(17.5-21.7)$ & $14.0-27.6$ \\
\hline Hip fracture type: cervical/trochanteric & N/A & $21 / 36$ & \\
\hline Grip strength of dominant arm at admission (kg) & N/A & $12.3(7.5-16.9)$ & $0-38.7$ \\
\hline Grip strength of female at admission $(\mathrm{kg})(\mathrm{n}=45)$ & N/A & $10.4(6.9-14.5)$ & $0-26.6$ \\
\hline Grip strength of male at admission $(\mathrm{kg})(\mathrm{n}=12)$ & N/A & $22.9(17.3-25.8)$ & $9.7-38.7$ \\
\hline HDS-R at admission & N/A & $14(5-22.5)$ & $0-30$ \\
\hline Hemoglobin $(\mathrm{g} / \mathrm{dL})$ & $11.5-15.0$ & $11.6(10.6-12.7)$ & 7.1-14.7 \\
\hline Albumin (g/dL) & $3.8-5.3$ & $3.4(3.0-3.9)$ & $2.2-4.2$ \\
\hline HbA1c $(\%)$ & 4.3-5.8 & $5.8(5.4-6.3)$ & $4.5-10.8$ \\
\hline Bone mineral density spine $\left(\mathrm{g} / \mathrm{cm}^{2}\right)$ & N/A & $0.69(0.60-0.81)$ & $0.37-1.09$ \\
\hline Isometric knee extension strength of healthy side on discharge (kgf) & N/A & $5.9(0-10.35)$ & $0.0-27.8$ \\
\hline Period of hospitalization & $\mathrm{N} / \mathrm{A}$ & $65(36.5-102.5)$ & $22-135$ \\
\hline
\end{tabular}

Note. Data are expressed as median (interquartile range (IQR)), range, or number.

Abbreviations: HbA1c, Hemoglobin A1c; HDS-R, Hasegawa's Dementia Scale-Revised; N/A, not applicable.

TABLE 2.

Correlation of factors to grip strength

\begin{tabular}{lcc}
\hline & $\mathrm{r}$ & $\mathrm{P}$ \\
\hline Age & -0.4097 & 0.0016 \\
Height $(\mathrm{m})$ & 0.6658 & $<.0001$ \\
Weight $(\mathrm{kg})$ & 0.4036 & 0.0019 \\
Body mass index $\left(\mathrm{kg} / \mathrm{m}^{2}\right)$ & 0.0687 & 0.6118 \\
Hemoglobin $(\mathrm{g} / \mathrm{dL})$ & 0.3046 & 0.0238 \\
Albumin $(\mathrm{g} / \mathrm{dL})$ & 0.1468 & 0.2848 \\
HbA1c $(\%)$ & 0.2362 & 0.0797 \\
HDS-R at admission & 0.5969 & $<.0001$ \\
Bone mineral density spine $\left(\mathrm{g} / \mathrm{cm}^{2}\right)$ & 0.4122 & 0.0018 \\
Isometric knee extension strength at & 0.6273 & $<.0001$ \\
healthy side at discharge $(\mathrm{kgf})$ & 0.2979 & 0.0244 \\
Period of hospitalization & 0.6232 & $<.0001$ \\
\hline FIM on discharge & &
\end{tabular}

TABLE 3.

Multivariate stepwise analysis for factors associated with walking acquisition

\begin{tabular}{lcccc}
\hline Factors & Unit & $\begin{array}{c}\text { Odds } \\
\text { ratio }\end{array}$ & $\begin{array}{c}95 \% \\
\text { Confidence } \\
\text { interval }\end{array}$ & P value \\
\hline Grip strength & 1 & 1.26 & $1.018-1.566$ & 0.0339 \\
HDS-R & 1 & 1.13 & $1.0123-1.257$ & 0.0188 \\
\hline
\end{tabular}

Abbreviations; HDS-R, Hasegawa's Dementia Scale-Revised.

Abbreviations: FIM, functional Independence Measure; HbA1c, Hemoglobin A1c; HDS-R, Hasegawa's Dementia Scale-Revised. 

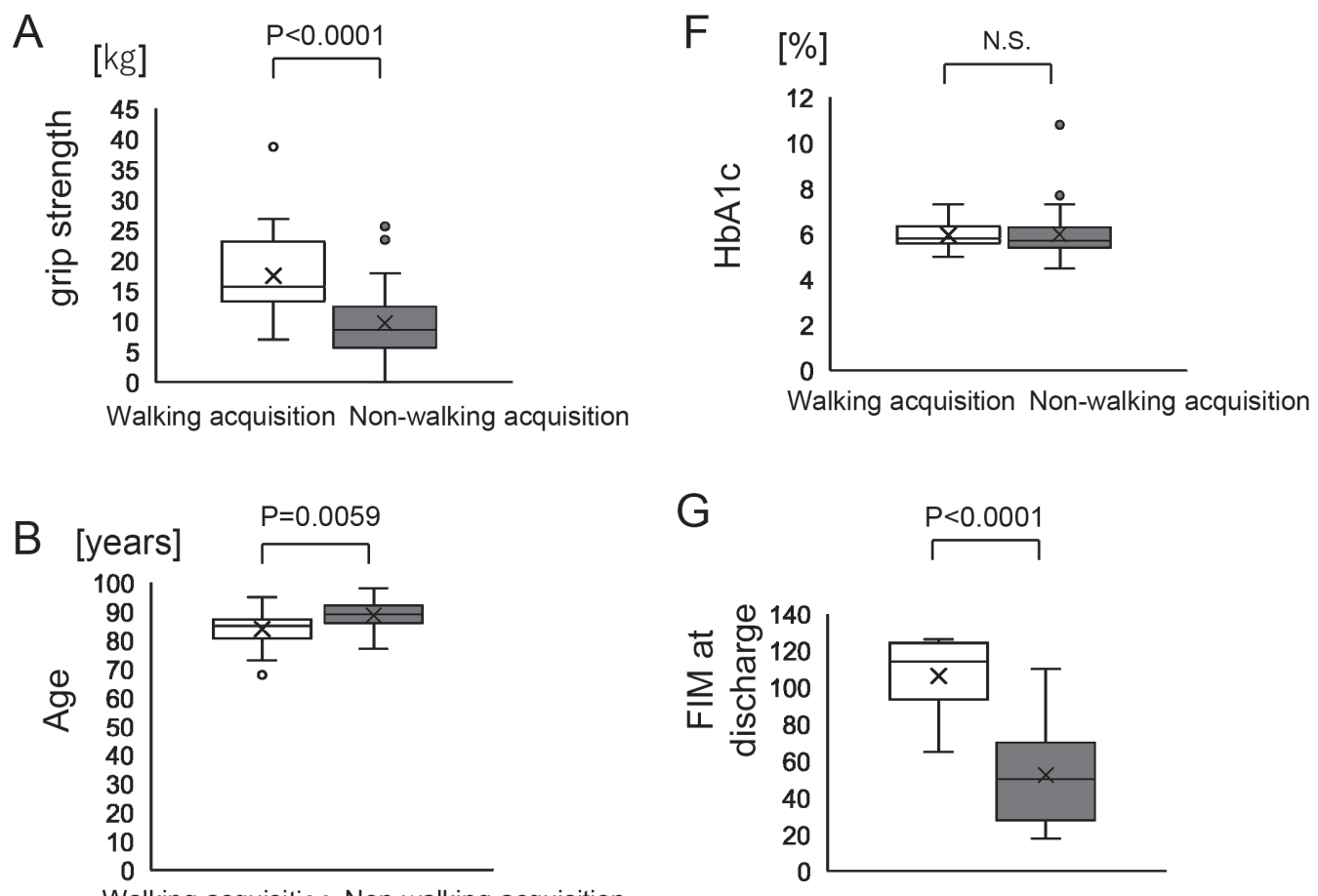

G

$$
\stackrel{P<0.0001}{\Gamma}
$$
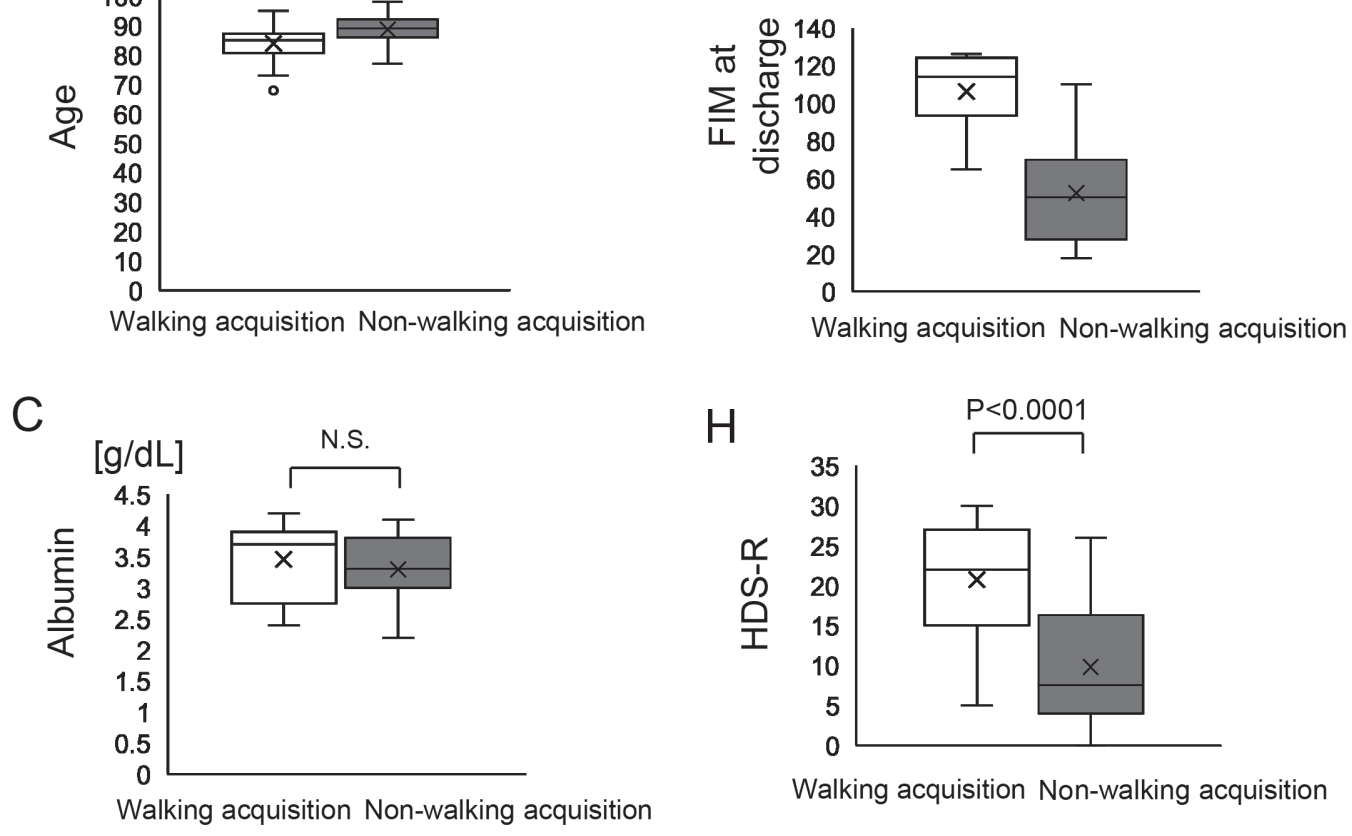

Walking acquisition Non-walking acquisition

D $\left[\mathrm{g} / \mathrm{cm}^{2}\right] \quad$ N.S.
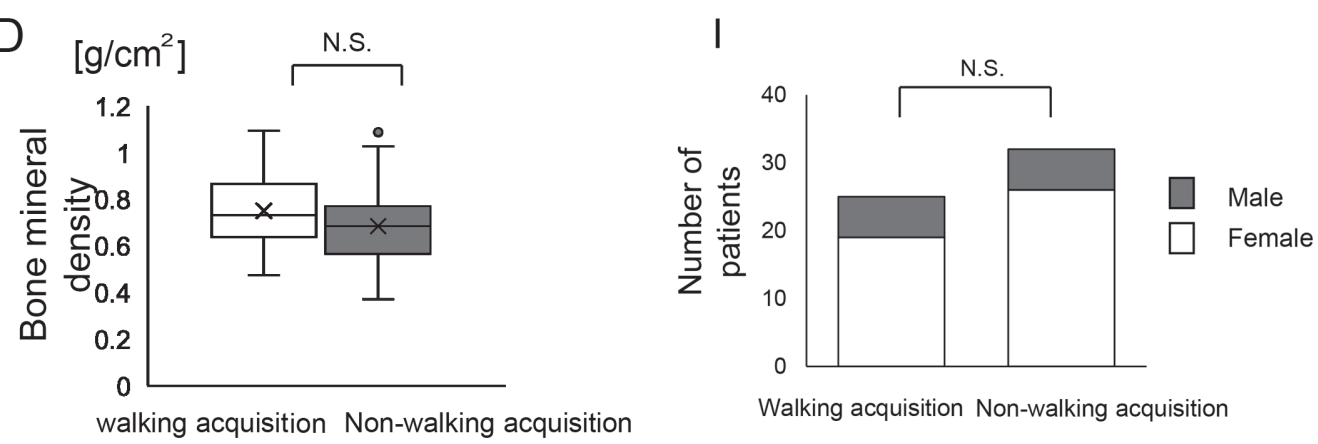

E
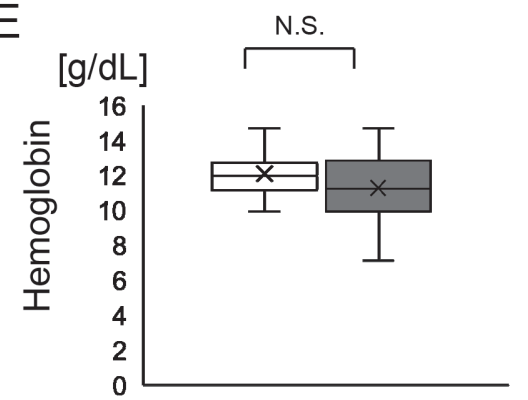

Fig. 1. Difference in grip strength, host factors, and FIM at discharge between the walking acquisition and non-walking acquisition groups Abbreviations; FIM, Functional Independence; HbA1c, Hemoglobin A1c; HDS-R, Hasegawa's Dementia Scale-Revised; N/A, not applicable.

Walking acquisition Non-walking acquisition 
with hand grip $\geqq 13.2 \mathrm{~kg}$ showing walking acquisition. For the patients with grip strength $<13.2 \mathrm{~kg}$, HDS-R was selected as the second split. Of the patients with grip strength $<13.2 \mathrm{~kg}$ and HDS-R $\geqq 15$, $45.5 \%$ showed walking acquisition (Figure 2 ).

\section{DISCUSSION}

In this study, we evaluated patients with hip fracture who had been admitted to a single hospital soon after fracture. Grip strength showed a positive correlation with isometric knee extension strength and FIM at discharge. In addition, grip strength was not only an independent factor for walking acquisition in the multivariate stepwise analysis, but was also an independent factor for walking acquisition in the decision-tree analysis. Thus, grip strength at admission is associated with walking ability at discharge. Grip strength is important for predicting the functional outcome of patients with hip fracture following surgery.

There are few well-validated techniques to measure muscle strength in clinical practice. Lower limbs are more relevant than upper limbs for gait and physical function. Lower limb strength would be useful for research studies; however, their use in clinical practice is limited by the need for special equipment and training [4]. On the other hand, handgrip strength is easily assessable and inexpensive. It is also useful as a diagnostic criterion for sarcopenia, as a substitute for lower limb muscle strength. Handgrip strength has been widely used and has been correlated with muscular strength and Activities of daily living (ADL) in various studies in the past $[14,15]$. Studies of the grip strength of hip fracture patients exist only for patients evaluated after admission to rehabilitation hospitals after surgery, and grip strength has been reported to show good functional prognosis [5,8]. Our study shows that grip strength at the time of acute stage hos- pital admission is not only positively correlated with isometric knee extension strength and FIM at discharge but that it was also an independent factor for walking acquisition. Because of the difficulty in measuring lower limb muscle strength immediately after fracture, grip strength is a useful tool, even in the acute phase.

One study reported that grip strength is useful for predicting the functional outcome of patients with hip fracture but that interventions to increase grip strength have not been adequately investigated [16]. Thirtyseven percent of patients with hip fracture experience sarcopenia. Sarcopenia increases the risk of falling, and nearly all hip fractures occur as the result of a fall [17]. Sarcopenia is a syndrome characterized by progressive and generalized loss of skeletal muscle mass and strength, with a risk of adverse outcomes such as physical disability, poor quality of life, or death $[8,18]$. Resistance exercise and nutritional intervention are recommended for patients with sarcopenia in order to improve muscle outcome $[18,19]$. Exercise interventions improve muscle strength and physical performance [18]. Moreover, nutrition improves muscle mass, prevents the onset of sarcopenia, and is associated with functional improvement in elderly patients with hip fractures [19]. Thus, appropriate exercise and nutritional intervention are thought to improve of the muscle disorder of hip fracture patients.

There are a few potential limitations in this study. We did not collect data on other potential confounders, including preinjury level of independence, nutritional state, prevalent vertebral fractures, depression, postoperative infection such as respiratory and urinary infection, or pain at various times during rehabilitation. There is wide variability in the protocols used for measuring grip strength, which can affect the resulting

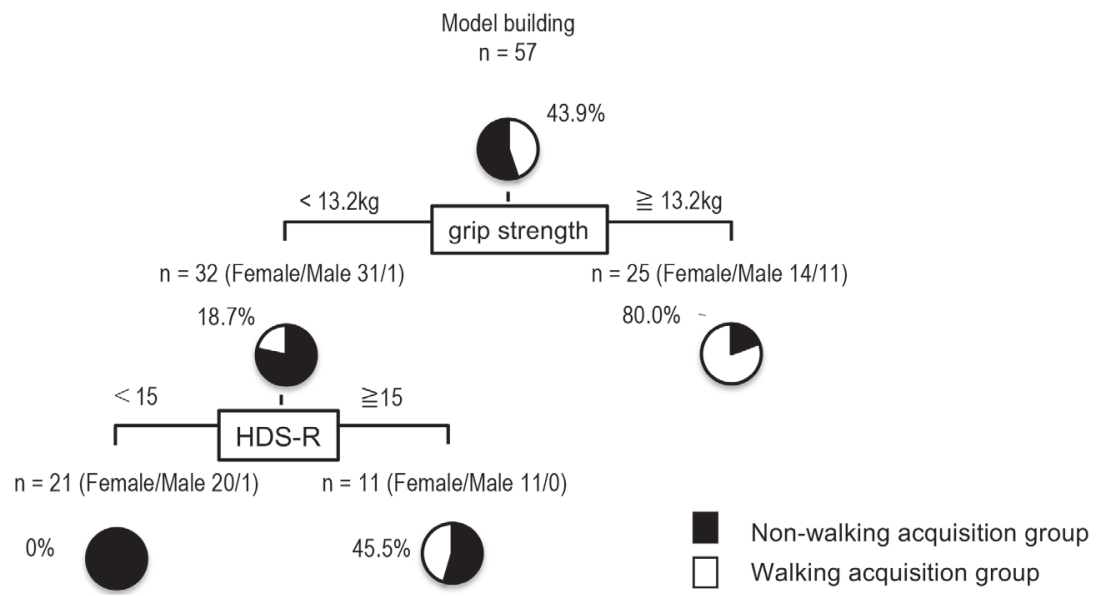

Kurume Medical Journal Vol. 66, No. 42019
Fig. 2. Factors related to walking acquisition Abbreviations; HDS-R, Hasegawa's Dementia Scale-Revised. 
values. The sample size in this study was small and it included both male and female patients. Because, the prevalence of hip fracture is higher in women, there were only 12 male patients in our study. The grip strength of men is generally higher than that of women. Consequently, our data cannot be generalized to the overall population of patients who sustain hip fractures. It will necessary to further investigate the difference between the sexes.

\section{CONCLUSIONS}

Grip strength is the only physical function that can be easily measured at the time of admission of patients with hip fracture. Grip strength at admission is strongly related to knee extension strength and the ADL of patients with hip fracture at discharge. Moreover, our data supports a prognostic role for grip strength on admission for patients after hip fracture. Grip strength would be a useful factor for predicting the future mobility of patients with hip fracture.

\section{CONFLICT OF INTEREST: None.}

AUTHORS' CONTRIBUTIONS: Ryuki Hashida, Hiroo Matsuse participated in study conception and design, interpretation of data, and drafting of the manuscript. Masafumi Bekki, Souhei Iwanaga, Takahiko Higuchi, Yohei Hirakawa, Asami Kubota, Hiromi Imagawa, Yoko Muta, Yumi Miruno, Kazuhito Minamitani, performed data acquisition. Naoto Shiba participated in critical revision.

ETHICS: This study's protocol conformed to the ethical guidelines of the Declaration of Helsinki as reflected in prior approval given by the institutional review board of Kurume University (Kurume, Japan, No 15019). Informed consent for participation in the study was obtained from each patient.

\section{REFERENCES}

1. Hirschfeld HP, Kinsella R, and Duque G. Osteosarcopenia: where bone, muscle, and fat collide. Osteoporos Int 2017; 28:2781-2790.

2. Burge R, Dawson-Hughes B, Solomon DH, Wong JB, King $\mathrm{A}$ et al. Incidence and Economic Burden of Osteoporosis-Related Fractures in the United States, 20052025. J Bone Miner Res 2007; 22:465-475.

3. Shi N, Foley K, Lenhart G, Badamgarav E. Direct healthcare costs of hip, vertebral, and non-hip, non-vertebral fractures. Bone 2009; 45:1084-1090.

4. Cruz-Jentoft AJ, Baeyens JP, Bauer JM, Boirie Y, Cederholm $\mathrm{T}$ et al. Sarcopenia: European consensus on definition and diagnosis: Report of the European Working Group on Sarcopenia in Older People. Age Ageing 2010; 39:412-423.

5. Di Monaco M, Castiglioni C, De Toma E, Gardin L,
Giordano $\mathrm{S}$ et al. Handgrip Strength is an Independent Predictor of Functional Outcome in Hip-Fracture Women: A Prospective Study With 6-Month Follow-Up. Medicine (Baltimore) 2015; 94:e542.

6. Norman K, Stobäus N, Gonzalez MC, Schulzke JD, and Pirlich M. Hand grip strength: Outcome predictor and marker of nutritional status. Clin Nutr 2011; 30:135-142.

7. Portegijs E, Sipilä S, Rantanen T, Lamb SE. Leg Extension Power Deficit and Mobility Limitation in Women Recovering from Hip Fracture. Am J Phys Med Rehabil 2008; 87:363-370.

8. Di Monaco M, Castiglioni C, De Toma E, Gardin L, Giordano et al. Handgrip Strength but not Appendicular Lean Mass is an Independent Predictor of Functional Outcome in Hip-Fracture Women: A Short-Term Prospective Study. Arch Phys Med Rehabil 2014; 95:17191724.

9. Hillman TE, Nunes QM, Hornby ST, Stanga Z, Neal KR et al. A practical posture for hand grip dynamometry in the clinical setting. Clin Nutr 2005; 24:224-228.

10. Suzuki T. Reliability of measurements of knee extensor muscle strength using a pull-type hand-held dynamometer. J Phys Ther Sci 2015; 27:967-971.

11. Keith RA, Granger CV, Hamilton BB, and Sherwin FS. The functional independence measure: a new tool for rehabilitation. Adv Clin Rehabil 1987; 1:6-18.

12. Kim KW, Lee DY, Jhoo JH, Youn JC, Suh YJ et al. Diagnostic Accuracy of Mini-Mental Status Examination and Revised Hasegawa Dementia Scale for Alzheimer's Disease. Dement Geriatr Cogn Disord 2005; 19:324-330.

13. Koya S, Kawaguchi T, Hashida R, Goto E, Matsuse H et al. Effects of in-hospital exercise on liver function, physical ability, and muscle mass during treatment of hepatoma in patients with chronic liver disease. Hepatol Res 2017; 47:E22-E34.

14. Rantanen T, Guralnik JM, Foley D, Masaki K, Leveille S et al. Midlife Hand Grip Strength as a Predictor of Old Age Disability. JAMA 1999; 281:558-560.

15. Lauretani F, Russo CR, Bandinelli S, Bartali B, Cavazzini $\mathrm{C}$ et al. Age-associated changes in skeletal muscles and their effect on mobility: an operational diagnosis of sarcopenia. J Appl Physiol 2003; 95:1851-1860.

16. Shyam Kumar AJ, Beresford-Cleary N, Kumar P, Barai A, Vasukutty $\mathrm{N}$ et al. Preoperative grip strength measurement and duration of hospital stay in patients undergoing total hip and knee arthroplasty. Eur J Orthop Surg Traumatol 2013; 23:553-556.

17. Steihaug OM, Gjesdal CG, Bogen B, Kristoffersen MH, Lien $\mathrm{G}$ et al. Sarcopenia in patients with hip fracture: A multicenter cross-sectional study. PLoS One 2017; 12:e0184780.

18. Cruz-Jentoft AJ, Landi F, Schneider SM, Zuniga C, Arai H et al. Prevalence of and interventions for sarcopenia in ageing adults: a systematic review. Report of the International Sarcopenia Initiative (EWGSOP and IWGS). Age Ageing 2014; 43:748-759.

19. Malafarina V, Uriz-Otano F, Malafarina C, Martinez JA, and Zulet MA. Effectiveness of nutritional supplementation on sarcopenia and recovery in hip fracture patients. A multi-centre randomized trial. Maturitas 2017; 101:42-50. 